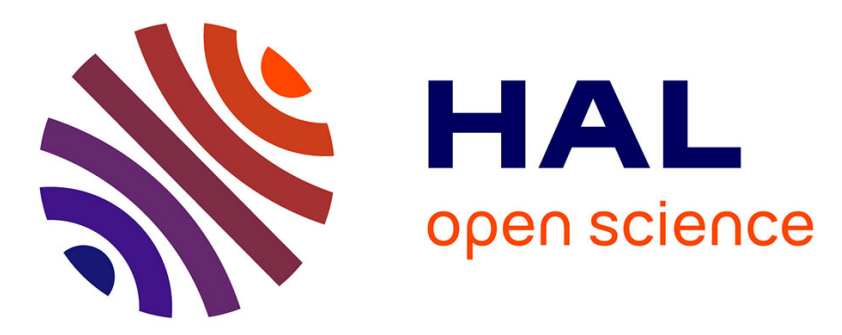

\title{
Photoluminescence observation of quantum confined stark effect caused by band bending near the surface of etched structures with GaAs/AlGaAs wells
}

\author{
V. Astratov, Yu. Vlasov
}

\section{To cite this version:}

V. Astratov, Yu. Vlasov. Photoluminescence observation of quantum confined stark effect caused by band bending near the surface of etched structures with GaAs/AlGaAs wells. Journal de Physique IV Proceedings, 1993, 03 (C5), pp.C5-277-C5-281. 10.1051/jp4:1993555 • jpa-00251641

\author{
HAL Id: jpa-00251641 \\ https://hal.science/jpa-00251641
}

Submitted on 1 Jan 1993

HAL is a multi-disciplinary open access archive for the deposit and dissemination of scientific research documents, whether they are published or not. The documents may come from teaching and research institutions in France or abroad, or from public or private research centers.
L'archive ouverte pluridisciplinaire HAL, est destinée au dépôt et à la diffusion de documents scientifiques de niveau recherche, publiés ou non, émanant des établissements d'enseignement et de recherche français ou étrangers, des laboratoires publics ou privés. 


\title{
Photoluminescence observation of quantum confined stark effect caused by band bending near the surface of etched structures with GaAs/AIGaAs wells
}

\author{
V.N. ASTRATOV and Yu.A. VLASOV \\ A.F. Ioffe Physical-Technical Institute, 194021 St. Petersburg, Russia
}

\begin{abstract}
The interaction of electronic and excitonic states confined in quantum wells (QW) with nearby sulphur-passivated surface was studied by photoluminescence (PL). The redshift (up to 12 meV for $50 \mathrm{~A} \mathrm{QW}$ ) and quenching (down to $10^{-4}$ ) of QW PL line were observed with approaching of the surface closely to $\mathrm{QW}$ by wet etching. The predominant role of quantum-confined Stark effect (QCSE) caused by the near-surface band bending was revealed by the following observations: (1) long-range surface influence $(>300 \mathrm{~A}),(2)$ increasing of the redshift for the thicker QW's, (3) the redshift dependence on the excitation level. On the basis of QCSE theory all the parameters of depleted layer on excitation level are determined. The revealed model can be used for the explanation of the optical properties of etched nanostructures.
\end{abstract}

\section{INTRODUCTION}

The interaction of states confined in quantum wells (QW) with nearby states at surfaces or interfaces play an important role in optical and electrical properties of a variety of nanometric structures and devices. It defines the speed parameters of the charge transfer based devices so as the radiative properties of laser structures. The surface properties essentially influence the radiative efficiency of low-dimensional systems, such as etched quantum wires and dots ${ }^{1-3}$.

Relatively new and promising possibility for the study of surface/well interaction gives the optical spectroscopy of near-surface QW's ${ }^{43}$. This method implies the variation of the distance from QW to the surface in nanoscale by epitaxial growth ${ }^{4-8}$ or etching ${ }^{9-13}$. The interaction with the surface is detected by PL originating from near-surface QW, which can be considered as a kind of local optical probe of the surface influence. In recent years this method was used for the study of the main III-V confined systems and a variety of surfaces. Several different models of surface/well interaction were suggested: (1) The mechanism of tunneling coupling of confined states with the surface states were proposed for the explanation of the inferaction with epitaxial and nonoxidized surface under high-vacuum in the system GaAs/AlGaAs ${ }^{4}$. This tunneling model was widely used also for the interpretation of surface/well interaction in different systems ${ }^{5-6}$. (2) The interaction with wet-etched and as-grown oxidized surfaces in InGaAs/GaAs system was explained by modeling of the surface as an abrupt termination of the structure with a quasiinfinite potential barrier 13 . (3) The studies of the interaction with dry-etched surface in GaAs/AlGaAs system revealed the deep-penetrated influence of the surface due to the formation of dry-etch-induced damages ${ }^{10}$.

The study of the interaction with passivated surfaces attract special interest, because different treatments can essentially reduce the density of surface states ${ }^{14}$. Spectroscopy of near-surface QW's may be used for characterization of the efficiency of varios surface passivation treatments 5,11 . However the mechanisms of the interaction with the passivated surfaces has not yet been well understood. Here we report on the PL measurements of the interaction of $\mathrm{GaAs} / \mathrm{Ga}_{0.3} \mathrm{Al}_{0.7} \mathrm{As} \mathrm{QW}$ with etched and sulphur-passivated surface. The predominant role of surface/well interaction mechanism caused by near-surface band bending and quantum-confined Stark effect (QCSE) is demonstrated (see also 12 ). 


\section{SAMPLE PREPARATION}

Multiple QW structures, used in this study, were grown by molecular beam epitaxy on semiinsulating GaAs (100) substrates. For experimental convenience a strongly nonuniform structures were selected. The widths of all QW's and barriers smoothly changed up to $3-4 \% / \mathrm{mm}$ due to variations in growth rate along the substrate plane, thus the structure had a 'wedge-like' geometry shown on the inset of Figure 1 . The average widths of QW's numbered opposite to the growth order were consequently: 1 - 13A, 2 - 20A, $3-30 \mathrm{~A}, 4-38 \mathrm{~A}, 5-53 \mathrm{~A}, 6-77 \mathrm{~A}, 7$ 136A. The $\mathrm{QW}$ 's were separated by $\mathrm{Al}_{0.3} \mathrm{Ga}_{0.7}$ As barriers with $200 \mathrm{~A}$ average width. The multi$\mathrm{QW}$ region was sandwiched between two $\mathrm{Al}_{0.4} \mathrm{Ga}_{0.6} \mathrm{As}$ layers $2200 \mathrm{~A}$ thick. In order to decrease the distance $d$ from $\mathrm{QW}$ to the surface the wet etching in $\mathrm{H}_{2} \mathrm{SO}_{4}: \mathrm{H}_{2} \mathrm{O}_{2}: \mathrm{H}_{2} \mathrm{O}$ (1:8:500) was used. The surface was passivated ${ }^{14}$ after etching for increasing the $\mathrm{PL}$ efficiency by depositing $\mathrm{Na}_{2} \mathrm{~S}$. During etching the surface was transferred to the depth $S$ deep into the structure being parallel to its initial plane and crossing different QW's.

\section{EXPERIMENTAL}

The distance $d$ can be easily varied in PL experiments by the shift of the position of excitation spot along the surface. Figure 1 presents the set of typical low-temperature $(T=2 K) P L$ spectra measured as a function of laser spot position $(a-e)$. The PL was excited by a focused beam of $488 \mathrm{~nm}$ line of an Ar-laser with excitation level $100 \mathrm{~W} / \mathrm{cm}^{2}$. Each spectrum consists of a series of excitonic peaks originating from QW's of different widths. Note that the lineshape of exciton PL was defined by exciton scattering on the interface roughness and was not influenced by variations of $\mathrm{QW}$ widths within the excitation spot. The exciton localization processes are insignificant as it was observed by comparison of PL and reflectance spectra measured from the same points. The PL lines 1-2 are not presented because they disappeared in spectra due to etching away of corresponding QW's. The main spectral effects of the surface influence are revealed on the line 3 originating from the nearest to the surface $\mathrm{QW}$ by comparison with PL spectra obtained before etching from the same points. These effects are the redshift and strong quenching of intensity of PL line with decreasing the distance $d$ from $a$ to $e$. Both effects can also be seen on PL line of the next from the surface $Q W 4$, but they are less pronounced. The PL line 5 as well as not presented PL lines 6 and 7 originated from deeply buried (>500A) and noninfluenced by the surface QW's display continuous blueshift from $a$ to $e$ due to 'wedge-like' geometry of the sample. It can be shown, that the changing of the distance $d$ between different positions of laser spot may be estimated as $\Delta d=S \cdot \Delta L / L$, where $\Delta \mathrm{L} / \mathrm{L}$ - the relative change in widths of deeply buried QW's 5-7 calculated from the spectral positions

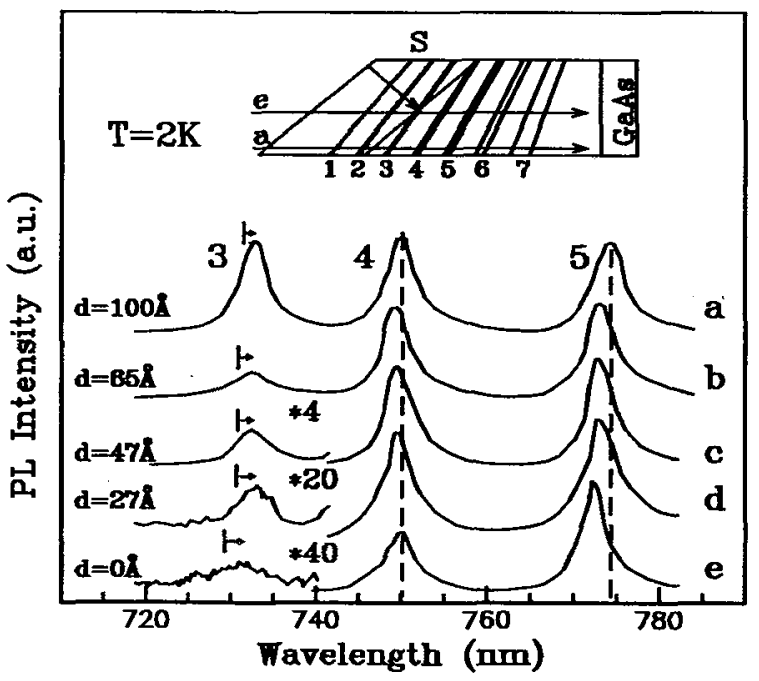

Fig.1. The set of low-temperature $(T=2 K)$ PL spectra measured as a function of sequential shift of excitation spot position (approximately $0.5 \mathrm{~mm}$ between points) along fixed direction on the sample plane. The arrows show the redshift of PL line from near-surface QW 3 measured from its spectral position in spectra obtained before etching from the same points. The calculated distances from near-surface $Q W 3$ to the etched surface are shown.

Inset: Schematic view of 'wedge-like' sample (not to scale). The etched part of the sample is shown by dotted lines. The etching depth $S$ is shown by the arrow. The arrows marked $a, e$ represent different positions of excitation spot corresponding to different spectra.

of their PL lines. As a reference point with zero distance from $\mathrm{QW}$ to the surface the position of 
laser spot was chosen corresponding to spectrum with PL line of near-surface. QW quenched down to $10^{-3}-10^{-4}$ relative to that in spectrum before etching. The value $d$ was calculated for the different spectra in relation with this reference point with the error less than $20 \mathrm{~A}$.

The observed effects of the surface influence on PL line of near-surface QW's with different widths are presented in Figure 2. The following peculiarities should be noted: the large distances $(>300 \mathrm{~A})$ of the interaction with the surface and well pronounced increase of the redshift for the thicker QW's.

\section{DICUSSION}

The main peculiarities of our experiment can not be explained by the tunneling coupling model $^{4}$, which is characterized by smaller interaction distances determined by the tunneling length $(\sim 100 \mathrm{~A})$ and by opposite dependence of the redshift on QW width. Note also, that observed effects are disagreed with the model $^{13}$ of quasi-infinite barrier at the surface of the structure, because it leads to the blueshift of near-surface QW PL line in opposition to the observed behavior.

We interpret the experimental data in the framework of the mechanism, based on band bending and QCSE. Near semiconductor surface the built-in electric field exists due to pinning of the Fermi level at the energetic position of charged surface states. The electric field in depleted layer distorts the $\mathrm{QW}$ potential that leads to the redshift of PL line of near-surface $\mathrm{QW}$ due to $\mathrm{QCSE}^{15}$. Therefore the surface/well interaction in this mechanism expands on distances determined by the Schottky length reaching under photoexcitation several hundreds of angstroms ${ }^{16}$ that is significantly larger than characteristic tunneling lengths and is in accordance with observed interaction distances. This mechanism can also describe the behavior of the redshift on $\mathrm{QW}$ width due to $\mathrm{QCSE}$ dependence $\triangle \mathrm{E} \sim \mathrm{L}^{4}$. Moreover the influence of built-in field on near-surface $\mathrm{QW}$ can also explain the effect of PL quenching by the depletion of near-surface region as well as by the field induced tunneling of carriers out of the QW shown on the inset of Figure 3. Note, that the latter process is sufficient for the explanation of the observed magnitude of quenching (similar values of quenching due to field induced tunneling were observed in experiments $^{17}$ with external electric fields

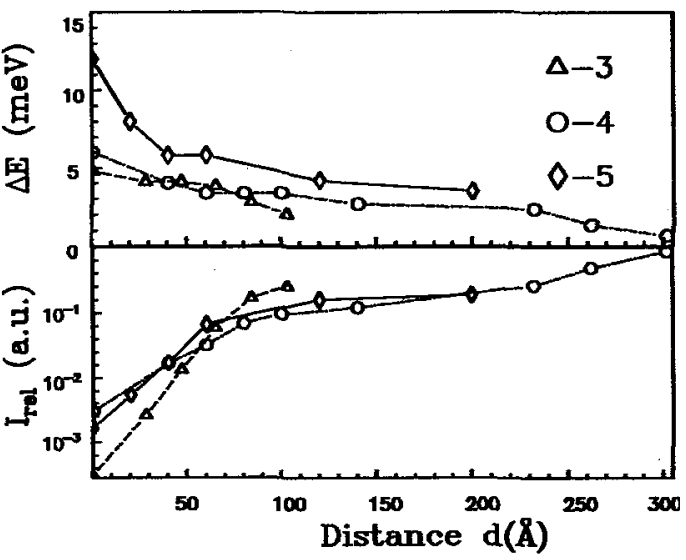

Fig.2. The redshift $\triangle E$ (upper plot) and relative intensity Irel (down plot) of PL line of near-surface $Q W ' s$ as a function of the distance from the surface $d$.

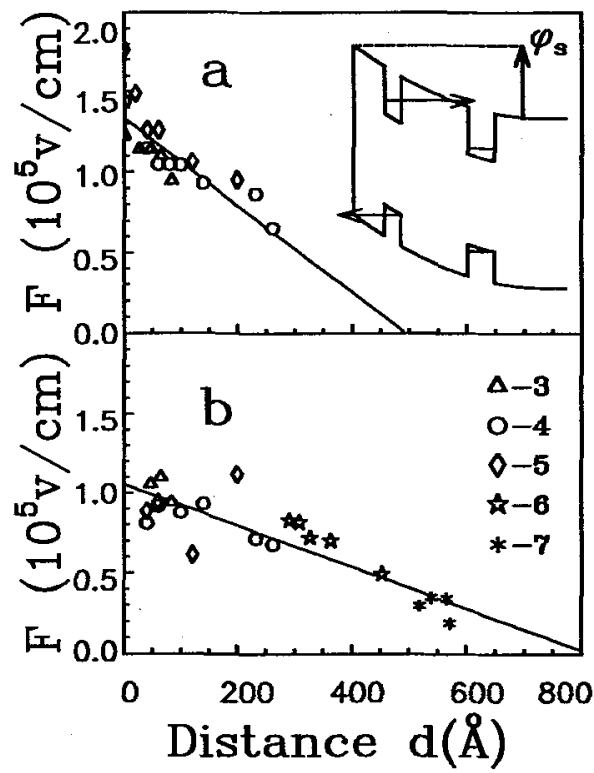

Fig.3. The near-surface electric field distributions calculated from the redshift data as a function of the distance $d$ from the surface at excitation levels (a) $100 \mathrm{~W} / \mathrm{cm}^{2}$ and (b) $\sim 4 \mathrm{~W} / \mathrm{cm}^{2}$.

Inset: $Q W$ 's with different widths in near-surface space-charge region. The arrows show the field induced tunneling of carriers out of the QW distorted by the $\sim 10^{5} \mathrm{~V} / \mathrm{cm}$ ). 
The electric field distributions were calculated directly from the redshift data $\Delta \mathrm{E}(d)$ by modeling the QCSE within the variational approach ${ }^{15}$ (Fig.3, a). Under photoexcitation $100 \mathrm{~W} / \mathrm{cm}^{2}$ the band bending $\varphi_{\mathrm{S}}=0.35 \mathrm{eV}$ is lowered in comparison with the dark value ${ }^{18}$ $\varphi_{\mathrm{S}}=0.9 \mathrm{eV}$ that displays the band flattening effect ${ }^{14,19}$. Space charge $N$ reaches $2 \cdot 10^{17} \mathrm{~cm}^{-3}$ essentially exceeding the residual doping by shallow impurities $\left(<10^{15} \mathrm{~cm}^{-3}\right)$ in the grown structure, that gives evidence for the processes of photoionization of deep levels in the barrier material. The latter is confirmed by the additional experiments performed with lower excitation level $4 \mathrm{~W} / \mathrm{cm}^{2}$. The calculated electric field distribution (Fig.3, b) obtained by the similar way from the redshift data is characterized by the values of screening parameters $\varphi_{\mathrm{S}}=0.43 \mathrm{eV}$, $N=9 \cdot 10^{16} \mathrm{~cm}^{-3}$ intermediate between the higher excitation case and corresponding dark values.

\section{CONCLUSION}

In conclusion it should be noted, that obtained data on near-surface field screening are of interest for the study of radiative properties of engraved nanostructures, such as etched quantum wires and dots ${ }^{1-3}$. According to this paper in these objects the strong electric field $\sim 10^{5} \mathrm{~V} / \mathrm{cm}$ parallel to QW plane should be expected. Such built-in electric field may be responsible for the formation of optically inactive 'dead' layer', which was suggested for explanation of PL quenching in ultrasmall nanostructures. Note also that the parameters of depleted layer may be controlled by photoexcitation conditions and, naturally, by different surface treatments that allow to modify the radiative properties of nanostructures. So the increase of PL efficiency of etched quantum wires with increasing of excitation density 3 may be explained not only by the saturation of the sidewall nonradiative recombination centers, but also by the band flattening effect accompanying with the narrowing of depleted layer.

We would like to thank A. A. Kaplyanskii for advices and encouragement.

\section{REFERENCES}

${ }^{1}$ B.E.Maile, A.Forchel and R.Germann, Appl.Phys.Lett. 54, 1552 (1989).

2 P.D.Wang, C.M.Sotomayor Torres, H.Benisty, C.Weisbuch and S.P.Beaumont Appl.Phys.Lett. 61, 946 (1992).

${ }^{3}$ H.Zull, B.Jacobs, A.Forchel, I.Gyuro, P.Speier and E.Zielinski, 13th General Conference CMD EPS, Regensburg, Germany, (European Conference Abstracts 17A, 1350 (1993)).

${ }^{4}$ J.-M.Moison, K.Elcess, F.Houzay, J.-Y.Marzin, J.-M. Gerard, F.Barthe and M.Bensoussan, Phys.Rev. B 41, 12945 (1990).

${ }^{5}$ F.Houzay, J.-M.Moison, K.Elcess and F.Barthe, Superlatt. and Microstr.,9,507 (1991).

${ }^{6}$ Z.Sobisiersky and D.I.Westwood, Superlatt. and Microstruct. 12, 267 (1992).

${ }^{7}$ R.M.Cohen, M.Kitamura and Z.M.Fang, Appl.Phys.Lett. 50, 1675 (1987).

8 Z.Sobisiersky, S.A.Clark, R.H.Williams, A.Tabata, T.Benyatton, G.Guillot, M.Gendry, G.Hollinger and P.Victorovich, Appl.Phys.Lett. 58, 1863 (1991).

${ }^{9}$ E.Yablonowitch, H.M.Cox and T.G.Gmitter, Appl.Phys.Lett. 52, 1002 (1988)

${ }^{10}$ D.L.Green, J.A.Skidmore, D.G.Lishan, E.L.Hu and P.M.Petroff, Appl.Phys.Lett. 62, 1253 (1993).

${ }^{11}$ Y.L.Chang, I.H.Tan, Y.H. Zhang, J.Merz and E.Hu, Appl.Phys.Lett., 62, 2697 (1993).

${ }^{12}$ V.N.Astratov and Yu.A.Vlasov, Fizika i Tehnika Poluprovodn., 27, 1101 (1993) (Semiconductors (former Sov.Phys.Semicond.) 27 1993)

13 J.Dreybrodt, A.Forchel and J.P.Reithmaier, Proceedings of OECS 3 Conference, Montpellier, France: Journal de Physique - Colloque (this issue).

${ }^{14}$ E.Yablonowitch, C.J.Sandroff, R.Bhat and T.G.Gmitter, Appl.Phys.Lett. 51, 439 (1987).

${ }^{15}$ G.Bastard, E.E.Mendez and L.Esaki, Phys.Rev. B 28, 3241 (1983). 
${ }^{16}$ E.Yablonowitch, B.J.Skromme, R.Bhat, J.P.Harbison and T.J.Gmitter, Appl.Phys.Lett. 54, 555 (1989).

${ }^{17}$ Y.Horicoshi, A.Fisher and K.Ploog, Phys.Rev. B 31, 7859 (1985).

${ }^{18}$ H.H.Weider, J.Vac.Sci.Technol. 17, 1009 (1980).

${ }^{19}$ D.Richards, J.Wagner and K.Ploog, Appl.Phys.Lett. 61, 2685 (1992). 